
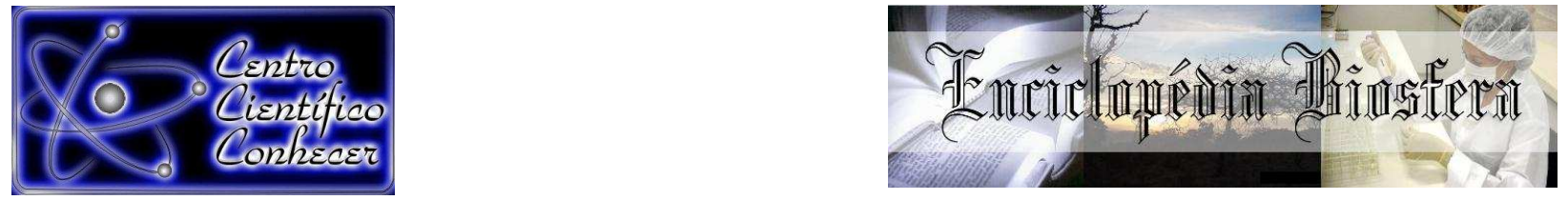

\title{
CARACTERIZAÇÃO BIOMÉTRICA DE FRUTOS E SEMENTES DE CASTANHA- DO-BRASIL NA AMAZÔNIA MATO-GROSSENSE
}

Vinicius Delgado da Rocha ${ }^{1}$, Joameson dos Santos Lima², Rosieli Barboza Bispo ${ }^{3}$, Jakeline Santos Cochev ${ }^{4}$, Ana Aparecida Bandini Rossi ${ }^{5}$

${ }^{1}$ Graduando do curso de Ciências Biológicas da Universidade do Estado do Mato Grosso - UNEMAT. Campus de Alta Floresta, MT. E-mail: viniciusdelgado123@hotmail.com

${ }^{2}$ Graduando do curso de Engenharia Florestal da Universidade do Estado do Mato

Grosso - UNEMAT. Campus de Alta Floresta, MT.

${ }^{3}$ Licenciada em Ciências Biológica pela Universidade do Estado do Mato Grosso UNEMAT. Campus de Alta Floresta, MT

${ }^{4}$ Doutoranda em Biodiversidade e Biotecnologia pela Rede Bionorte, Universidade do Estado de Mato Grosso - UNEMAT. Campus de Alta Floresta, MT.

${ }^{5}$ Professora Doutora da Faculdade de Ciências Biológicas e Agrárias. Universidade do Estado do Mato Grosso - UNEMAT. Campus de Alta Floresta, MT, Brasil.

Recebido em: 03/10/2016 - Aprovado em: 21/11/2016 - Publicado em: 05/12/2016 DOI: 10.18677/EnciBio_2016B_017

RESUMO
Bertholletia excelsa Bonpl. (castanha-do-brasil) é uma espécie arbórea com ocorrência na Amazônia, sendo seus frutos e sementes comercializados nacionalmente e internacionalmente. A biometria de frutos é uma importante ferramenta para avaliar a variabilidade genética dentro de populações de uma mesma espécie, além disso, pode contribuir para a tecnologia de produção de mudas de espécies nativas. Diante do exposto, objetivou-se caracterizar fisicamente frutos e sementes de árvores de $B$. excelsa, ocorrentes em Alta Floresta, MT. Foi realizada caracterização de 40 frutos e 677 sementes provenientes de doze indivíduos de $B$. excelsa. Foram avaliadas as seguintes características: comprimento longitudinal, largura, massa, espessura da semente e número de sementes por fruto. Os dados foram analisados utilizando medidas descritivas: distribuição de frequência, média aritmética, valor mínimo e máximo, variância, desvio padrão, coeficiente de variação, e pelo coeficiente de correlação de Spearman (rS). Para os frutos, o comprimento longitudinal, largura, massa e número de sementes tiveram média de 10,72 cm 11,26 cm, 425,27 g e 16,92, respectivamente. Já para as sementes, o comprimento longitudinal, largura, espessura e massa tiveram média de $4,23 \mathrm{~cm}, 2,67 \mathrm{~cm}, 1,93 \mathrm{~cm}$ e $8,17 \mathrm{~g}$, respectivamente. Notou-se uma ampla distribuição de frequência das características avaliadas nos frutos e nas sementes. Houve grande variação nas características biométricas de frutos e sementes de $B$. excelsa, principalmente em relação á massa. A alta variação fenotípica encontrada nas características avaliadas demonstra além das influências ambientais, a existência de variabilidade genética, relevando assim o potencial para conservação de germoplasma e melhoramento genético desta espécie.

PALAVRAS-CHAVE: Árvores nativas; Bertholletia excelsa; variabilidade. 


\title{
BIOMETRIC CHARACTERIZATION OF FRUIT AND SEEDS OF BRAZIL NUT IN THE AMAZON MATO GROSSO
}

\begin{abstract}
Bertholletia excelsa Bonpl. (Brazil nut) is a tree species with occurring in the Amazon, its fruits and seeds are commercialized nationally and internationally. Biometry of fruit is an important resource for evaluate the genetic variability within populations of the same species, furthermore, it can contribute to the production technology of seedlings of native species. This study aimed to characterize fruits and seeds of trees B. excelsa, with occurring in Alta Floresta, MT. We performed the characterization of 40 fruits and 677 seeds from twelve individuals of $B$. excelsa. The characteristics evaluated were: longitudinal length, width, mass, thickness of seed and number of seeds per fruit. Data were analyzed using descriptive statistics: frequency distribution, arithmetic average, minimum and maximum value, variance, standard deviation, coefficient of variation and by Spearman correlation coefficient ( $r S$ ). For fruit the longitudinal length, width, mass and number of seeds had an average of $10.72 \mathrm{~cm} 11.26 \mathrm{~cm}, 425.27 \mathrm{~g}$ and 16.92 , respectively. For the seeds the longitudinal length, width, thickness and mass had an average of $4.23 \mathrm{~cm}, 2.67 \mathrm{~cm}, 1.93 \mathrm{~cm}$ and $8.17 \mathrm{~g}$, respectively. One large frequency distribution was noted in the characteristics evaluated in fruits and seeds. There was wide variation in biometric characteristics of fruit and seeds of $B$. excelsa, mainly in relation to mass. The high phenotypic variation observed in the evaluated characteristics demonstrates environmental influences as well as existence of genetic variability, which reveals the potential for conservation of germplasm and breeding of this species.
\end{abstract}

KEYWORDS: native trees; Bertholletia excelsa; variability

\section{INTRODUÇÃO}

A castanha-do-brasil (Bertholletia excelsa Bonpl.) é uma espécie pertencente à família Lecythidaceae com ocorrência na Amazônia, sendo um importante recurso para populações locais (SHEPARD JR \& RAMIREZ, 2011). Trata-se uma árvore semidecídua, heliófila, característica da mata alta de terra firme, podendo chegar excepcionalmente á 60 metros de altura, com tronco retilíneo de 100 a $180 \mathrm{~cm}$ de diâmetro (LORENZI, 2000).

O extrativismo de produtos florestais não madeireiros da castanha-do-brasil é uma das principais fontes de rendimento para os grupos indígenas da Bolívia, Brasil e Peru (ANGELO et al., 2013). Segundo BOUVIE et al. (2016), após a decadência da borracha, a castanha-do-brasil se tornou o principal símbolo do desenvolvimento sustentável e estratégico para conservação da floresta Amazônica. Da castanha-dobrasil, coletam-se frutos para obtenção de sementes ou amêndoas que são nutritivas e ricas em selênio, sendo comercializadas no mercado nacional e internacional. Essas amêndoas são consumidas in natura, na forma de subprodutos ou podem ser utilizadas para extração de óleo empregado na produção de cosméticos (FREITAS et al., 2007).

No entanto, com a degradação da floresta amazônica e a contínua fragmentação dos habitas, a maioria dos castanhais tem se reduzido devido à construção de rodovias e ferrovias, deslocamento de migrantes, obras de infraestrutura, criação de novos municípios, expansão da atividade agropecuária e extração de madeira (CAMARGO et al., 2010). A castanha-do-brasil é uma espécie alógama com síndrome de polinização melitófila, necessitando da atividade de 
polinizadores para assegurar a produção de frutos, seus principais polinizadores são abelhas médias e grandes (MAUÉS, 2002; CAMPOS et al,. 2013). Porém a destruição do habitat natural dos agentes polinizadores tem contribuído para a diminuição da produção das castanheiras.

Considerando a importância econômica, social e ambiental da castanheira, análises de caracterização de frutos e sementes tornam-se necessárias, pois estudos de biometria de frutos podem fornecer informações úteis para conservação e exploração de recursos de valor econômico, permitindo um incremento contínuo da busca racional e uso eficaz dos frutos (GUSMÃO et al., 2006). Além disso, a biometria de frutos é uma importante ferramenta utilizada para avaliar a variabilidade genética dentro de populações de uma mesma espécie, e as relações desta variabilidade com fatores ambientais, fornecendo maior conhecimento sobre aspectos ecológicos (MACEDO et al., 2009).

Neste contexto, objetivou-se caracterizar frutos e sementes de árvores de $B$. excelsa, ocorrentes em Alta Floresta, MT, visando verificar a variabilidade genética intraespecífica e determinar características que potencializem as coletas de frutos com maior número de sementes no campo.

\section{MATERIAL E MÉTODOS}

Foram coletados frutos provenientes de doze indivíduos $B$. excelsa, com ocorrência natural em bordas ou dentro de fragmentos florestais, localizados no período urbano do município de Alta Floresta, Mato Grosso (Figura 01).

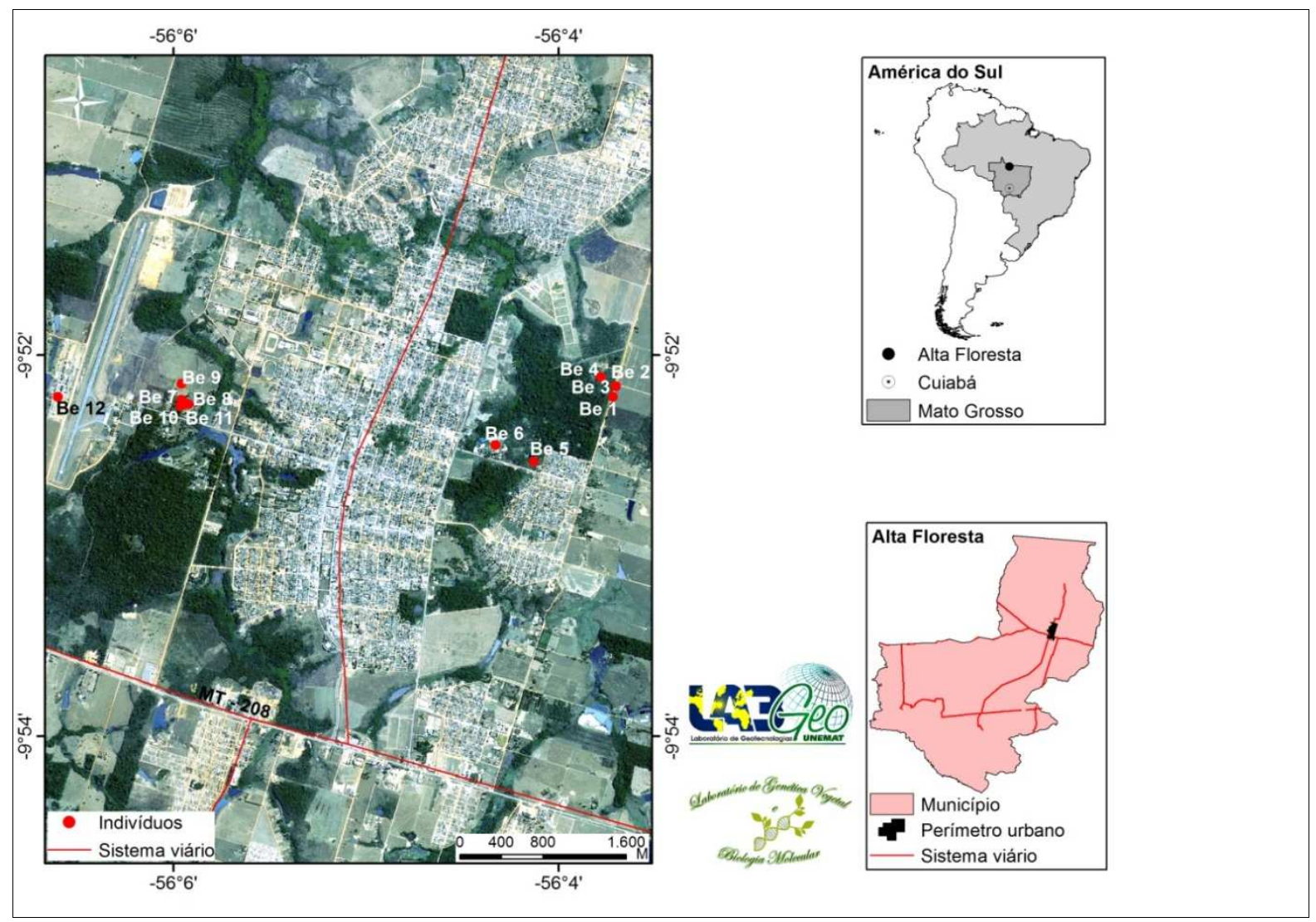

FIGURA 1. Localização dos indivíduos de $B$. excelsa no perímetro urbano do município de Alta Floresta, MT. 
Segundo KOPPEN (1948), o clima da região é do tipo AWI, com nítida estação seca e com temperaturas entre 20 a $38^{\circ} \mathrm{C}$, tendo em média $26 \stackrel{\circ}{\circ}$. A cobertura vegetal dominante é do tipo Floresta Ombrofila Aberta Tropical (BUTTURI et al., 2013). Para realização deste estudo, no período de frutificação entre os meses de janeiro a fevereiro de 2014 , foram coletados quarenta frutos de $B$. excelsa, dos quais foram avaliadas 677 sementes. Os frutos foram devidamente identificados e armazenados em sacos plásticos em campo, sendo posteriormente conduzidos ao laboratório.

De cada fruto determinou-se a massa fresca (MFF) em balança digital, e o comprimento longitudinal (CLF) e a largura (LF), com auxilio de um paquímetro digital. Considerou-se como largura, o diâmetro transversal. Os frutos foram seccionados para remoção das sementes, posteriormente contabilizou-se o número de sementes por fruto (NSF). Em cada semente foram avaliadas as seguintes características biométricas: comprimento longitudinal (CLS), largura mediana (LS), espessura mediana (ES) e massa fresca (MFS).

Para os dados obtidos das variáveis analisadas, foram calculadas medidas descritivas: distribuição de frequência, média aritmética, valor mínimo e valor máximo, variância, desvio padrão e coeficiente de variação. Também, foi calculado o coeficiente de correlação não paramétrico de Spearman (rS).

\section{RESULTADOS E DISCUSSÃO}

Nas Tabelas 1 e 2 estão apresentados os dados obtidos para biometria dos frutos e sementes de $B$. excelsa. O comprimento do fruto variou de 8 a $13,20 \mathrm{~cm}$, com média de 10,72 cm, enquanto a largura apresentou valores de 8,50 a 13,50 cm, com média de $11,26 \mathrm{~cm}$. Observou-se grande variação na massa de fruto, sendo encontrado valor mínimo de $154,32 \mathrm{~g}$, valor máximo de $748,97 \mathrm{~g}$, e uma média de $425,27 \mathrm{~g}$.

Em relação ao número de sementes, os valores variaram de 10 a 25 , com média de 16,62 sementes por fruto (Tabela 1). O valor médio obtido para variável número de semente por fruto (NSF) neste estudo foi semelhante ao encontrado por CAMARGO et al. (2010) e por BORGES et al. (2016), ao avaliarem castanheiras nativas do estado de Mato Grosso.

TABELA 1. Comprimento longitudinal (CLF), largura (LF), massa fresca (MFF) e número de sementes (NSF) dos frutos de $B$. excelsa.

\begin{tabular}{lcccccc}
\hline Variáveis & Mínimo & Máximo & Média & Variância & DP & CV (\%) \\
\hline CLF $(\mathbf{c m})$ & 8,00 & 13,20 & 10,72 & 1,74 & 1,32 & 12,32 \\
LF $(\mathbf{c m})$ & 8,50 & 13,50 & 11,26 & 1,51 & 1,23 & 10,93 \\
MFF (g) & 154,32 & 748,97 & 425,27 & 21203,66 & 145,61 & 34,24 \\
NSF (um) & 10,00 & 25,00 & 16,92 & 12,07 & 3,47 & 20,53 \\
\hline
\end{tabular}

DP: desvio padrão; CV: coeficiente de variação

O comprimento e a largura das sementes variaram de 2 a 6,01 $\mathrm{cm} \mathrm{e} \mathrm{1,68} \mathrm{a}$ $3,95 \mathrm{~cm}$, respectivamente. Já para variável espessura os valores oscilaram de 1,14 a 3,27 cm, com média de $1,93 \mathrm{~cm}$. A massa das sementes apresentou valor mínimo de $1,50 \mathrm{~g}$, valor máximo de $14,89 \mathrm{~g}$ e uma média de $8,17 \mathrm{~g}$ (Tabela 2). TONINI et al. (2008) avaliaram sementes de populações $B$. excelsa localizadas no sul do estado de Roraima, e o valor médio da massa foi de $4,07 \mathrm{~g}$, ou seja, inferior ao encontrado neste estudo. Possivelmente essa diferença deve-se a variação das condições 
edafoclimáticas entre as áreas de coleta. Segundo BOTEZELLI et al. (2000) em cada localidade geográfica, as sementes estão sujeitas a variações de temperatura, comprimento do dia e índices de pluviosidade.

TABELA 2. Comprimento longitudinal (CLS), largura (LS), espessura (ES), massa fresca (MFS) das sementes de $B$. excelsa.

\begin{tabular}{lcccccc}
\hline Variáveis & Mínimo & Máximo & Média & Variância & DP & CV (\%) \\
\hline CLS (cm) & 2,00 & 6,01 & 4,23 & 32,84 & 5,73 & 13,55 \\
LS (cm) & 1,68 & 3,95 & 2,67 & 11,93 & 3,45 & 12,94 \\
ES (cm) & 1,14 & 3,27 & 1,93 & 8,56 & 2,92 & 15,13 \\
MFS (g) & 1,50 & 14,89 & 8,17 & 6,11 & 2,47 & 30,25 \\
\hline
\end{tabular}

DP: desvio padrão; CV: coeficiente de variação

Os valores de desvio padrão das características biométricas dos frutos e sementes foram relativamente altos, indicando grande variação entre as amostras, o que pode estar relacionado à variabilidade genética e/ou fatores ambientais não controlados, tais como a condição de antropização, o solo, o clima e a idade da planta (SILVA et al., 2001). Vários estudos têm mostrado que espécies arbóreas tropicais possuem alta variabilidade quanto ao tamanho do fruto, número de sementes e massa dos frutos (BRAGA et al., 2007; MATOS et al., 2014; ZUFFO et al., 2014).

Os resultados referentes à distribuição de frequência das características analisadas nos frutos estão apresentados na Figura 2. Para comprimento verificouse que a maioria dos frutos concentrou-se em duas classes que juntam representaram $55 \%$, que foram de 9,74 a $11,48 \mathrm{~cm}$ (Figura $2 \mathrm{~A}$ ). Em relação à largura dos frutos, houve duas classes com maior frequência, sendo elas: 10,18 a $11,02 \mathrm{~cm}(22,50 \%)$ e 11,86 a $12,70 \mathrm{~cm}(27,50 \%)$ (Figura 2B).

Para a variável massa do fruto (MFF) observou-se que $50 \%$ amostras apresentam valores entre 253,43 a $451,65 \mathrm{~g}$ (Figura 2C). Quanto ao número de sementes, $65 \%$ dos frutos possuem de 15 a 22,50 sementes (Figura 2D).

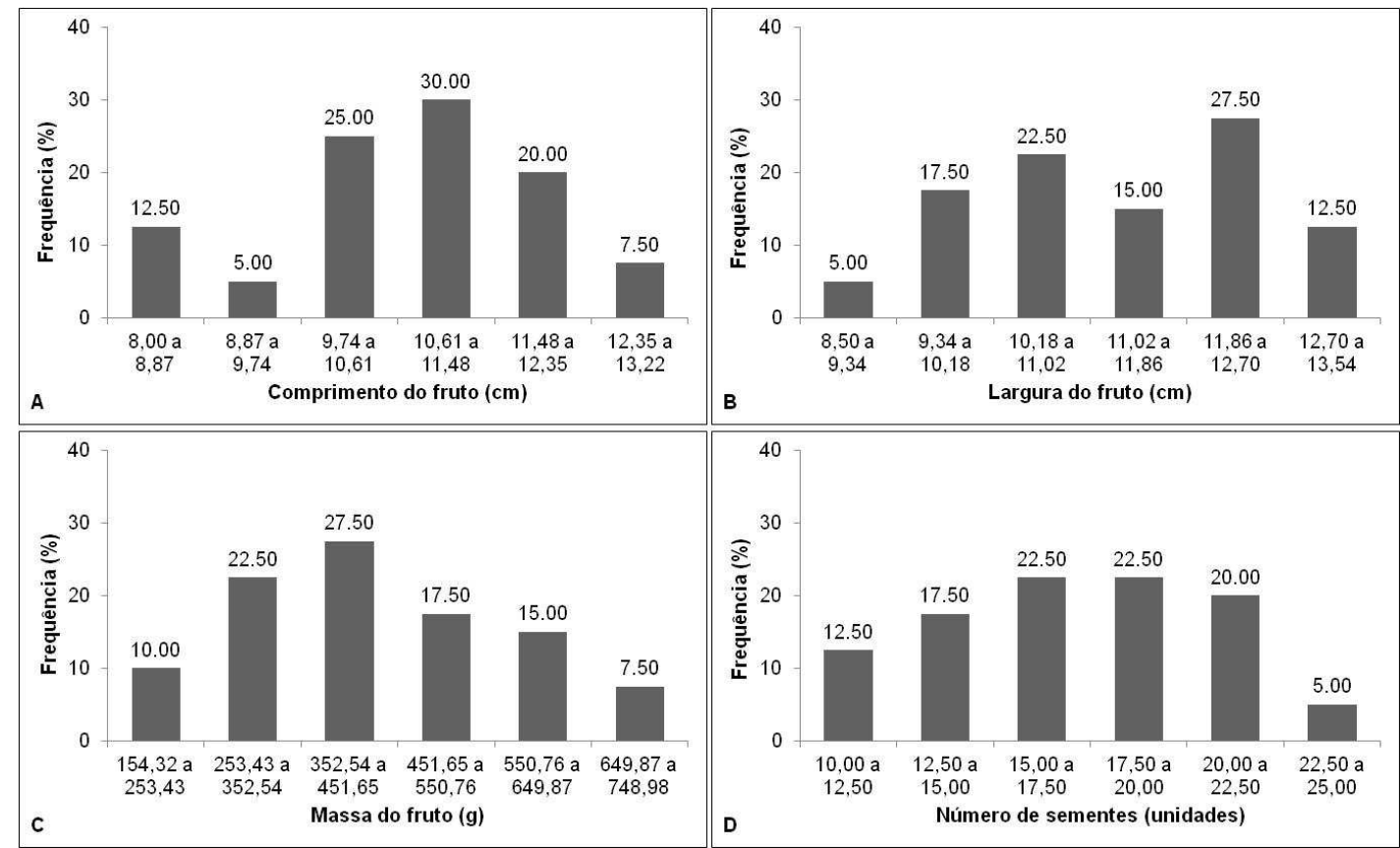

FIGURA 2. Frequência das características biométricas de frutos de $B$. excelsa. ENCICLOPÉDIA BIOSFERA, Centro Científico Conhecer - Goiânia, v.13 n.24; p.190 2016 
Na Figura 3 encontram-se os dados referentes à distribuição de frequência para características avaliadas em sementes de $B$. excelsa, onde nota-se que a maior parte das sementes $(44,02 \%)$ apresentou comprimento entre 4,01 a 4,68 cm (Figura $3 \mathrm{~A})$. Na variável largura $42,25 \%$ das sementes foram agrupadas na classe 2,44 a $2,82 \mathrm{~cm}$. Com relação á espessura das sementes foi possível determinar duas classes com maior frequência, que representaram $77,55 \%$, que foram de 1,50 a $2,22 \mathrm{~cm}$. Cerca de $60 \%$ das sementes concentrou a massa principalmente no intervalo de 5,98 a 10,46 g (Figura 3D).

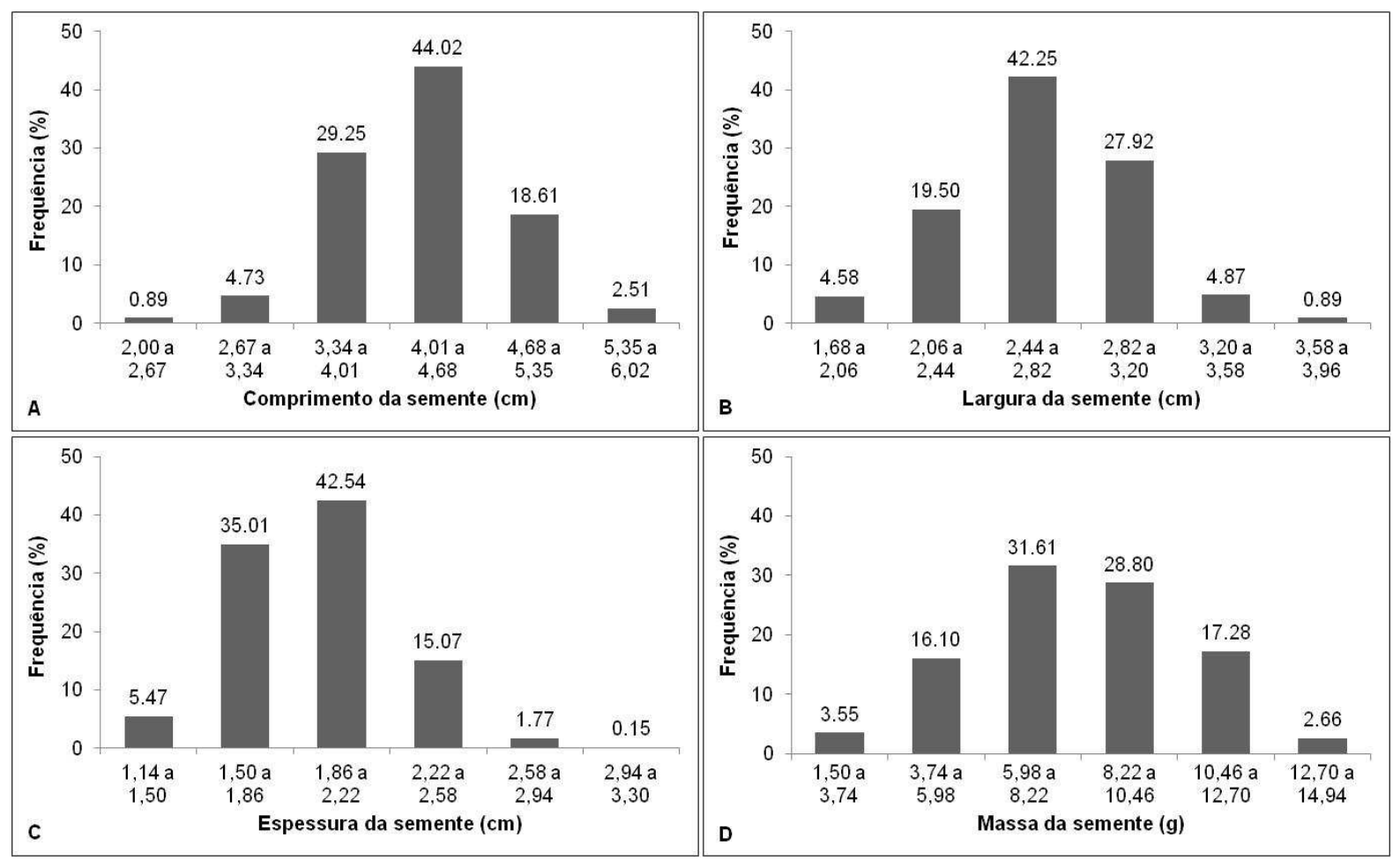

FIGURA 3. Frequência das características biométricas de sementes de $B$. excelsa.

O conhecimento da variação biométrica de características de frutos e sementes é útil para formação de bancos de germoplasma e para o melhoramento, pois essas informações podem ser exploradas em programas de melhoramento direcionados para geração de cultivares que produzam frutos com características que melhorem a sua comercialização (SANTOS et al., 2009; GONÇALVES et al., 2013).

Conforme as estimativas dos coeficientes de correlação de Spearman (rS) entre as características biométricas de frutos e sementes de castanheira (Tabela 1), constatou-se que o comprimento longitudinal do fruto (CLF) correlacionou-se positivamente com as demais variáveis, exceto com a espessura da semente (ES). A maior correlação foi entre largura do fruto (LF) e massa do fruto (MFF), indicando que quanto maior largura do fruto maior é a sua massa. Isso permite inferir que a seleção de frutos com maior massa pode ser feita diante da mensuração da largura, sendo relegada a necessidade de pesá-los, o que facilita a escolha de frutos com maior massa, ainda em campo.

A variável LF, também se correlacionou positivamente com NSF, CLS, LS, ES. Houve efeito das correlações para massa do fruto (MFF) com NSF, CLS, LS, ES e MFS. Assim, nas amostras de frutos e sementes de B. excelsa em análise, pode-se 
afirmar que quanto maior a massa de fruto, maior será a quantidade e o tamanho das sementes.

TABELA 3. Dados da correlação de Spearman ( $r S$ ) para as variáveis biométricas dos frutos e sementes de $B$. excelsa.

\begin{tabular}{|c|c|c|c|c|c|c|c|c|}
\hline & CLF & LF & MFF & NSF & CLS & LS & ES & MFS \\
\hline CLF & 1 & & & & & & & \\
\hline LF & $0,67^{* *}$ & 1 & & & & & & \\
\hline MFF & $0,69^{* *}$ & $0,94^{\star *}$ & 1 & & & & & \\
\hline NSF & $0,39^{* *}$ & $0,49^{* *}$ & $0,58^{* *}$ & 1 & & & & \\
\hline CLS & $0,57^{\star *}$ & $0,78^{* *}$ & $0,70^{* *}$ & $0,07^{\text {ns }}$ & 1 & & & \\
\hline LS & $0,35^{*}$ & $0,70^{* *}$ & $0,69^{* *}$ & $0,04^{\mathrm{ns}}$ & $0,63^{* *}$ & 1 & & \\
\hline ES & $0,25^{\text {ns }}$ & $0,67^{* *}$ & $0,68^{* *}$ & $0,09^{\text {ns }}$ & $0,68^{* *}$ & $0,79^{* *}$ & 1 & \\
\hline MFS & $0,42^{* *}$ & $0,81^{* *}$ & $0,79^{* *}$ & $0,16^{\text {ns }}$ & $0,83^{* *}$ & $0,88^{\star *}$ & $0,85^{\star *}$ & 1 \\
\hline
\end{tabular}

De acordo com FARIAS NETO et al. (2004), estimativas de correlação podem ser úteis quando determinada característica de interesse é de difícil avaliação, assim, o processo de seleção se torna mais simples, se essa característica apresentar alta correlação positiva com outra de fácil avaliação. Ou seja, aumentos em uma característica tendem estar associado a aumentos na outra e vice-versa, dessa maneira, não há necessidade de adoções de restrições no processo de seleção para obtenção de ganhos no sentido desejado.

A correlação positiva observada entre o tamanho do fruto (CLP e LF), massa do fruto e número de sementes por fruto é importante para populações extrativistas, pois a semente é o principal produto florestal não madeiro comercializado da castanha-do-brasil, sendo assim, o resultado encontrado permite inferir que a escolha de frutos com maior número de semente pode ser realizada diante da avaliação do tamanho do fruto no momento da coleta. Isso facilita o processo de seleção, já que o tamanho do fruto é mais fácil de ser avaliado em campo, do que o número de sementes.

\section{CONCLUSÃO}

Houve grande variação nas características biométricas de frutos e sementes de B. excelsa, principalmente em relação à massa. A alta variação fenotípica encontrada nas características avaliadas demonstra além das influências ambientais, a existência de variabilidade genética, relevando assim o potencial para conservação de germoplasma e melhoramento genético desta espécie.

\section{AGRADECIMENTOS}

Ao PROBIC/ Universidade do Estado de Mato Grosso (UNEMAT) pela concessão de bolsa ao primeiro e segundo autor. À FAPEMAT pelo apoio financeiro concedido ao projeto Análise da diversidade genética e distribuição espacial de genótipos em populações de Bertholletia excelsa Bonpl. por marcadores moleculares e anatômicos.

\section{REFERÊNCIAS}

ANGELO, H.; POMPERMAYER, R.S.; ALMEIDA, A.N.; MOREIRA, J.M.M.A.P. O custo social do desmatamento da Amazônia brasileira: o caso da castanha-do-brasil 
(Bertholletia excelsa). Ciência Florestal, v. 23, n. 1, p. 183-191, 2013. Disponível em: https://periodicos.ufsm.br/cienciaflorestal/article/view/8452/pdf_1. doi: $10.5902 / 198050988452$.

BORGES, F.A.; TONINI, H.; BALDONI, A. B.; BOTELHO, S.C.C. Tamanho da amostra para estimar produção de sementes de castanheiras nativas. Nativa, v.4, n.3, p.166-169, 2016. Disponível em: http://periodicoscientificos.ufmt.br/ ojs/index.php/nativa/article/ view/3429. doi: 10.14583/2318-7670.

BOTEZELLI, L.; DAVIDE, A.C.; MALAVASI., M.M. Características dos frutos e sementes de quatro procedências de Dipteryx alata Vogel (Baru). Cerne, v.6, n.1, p.9-18, 2000. Disponível em: http://www.redalyc.org/pdf/744/74460102.pdf.

BOUVIE, L.; BORELLA, D.R. PORTO, P.A.O.; SILVA, A.C, LEONEL, S. Caracterização físico-química dos frutos de castanheira do Brasil. Nativa, v.4, n.2, p.107-111, 2016. Disponível em: http://www.periodicoscientificos.ufmt.br/ ojs/index.php/nativa/article/view/3253/pdf. doi: 10.14583/2318-7670.

BRAGA, L.F.; SOUSA, M.P.; GILBERTI, S.; CARVALHO, M.A.C.; Caracterização morfométrica de sementes de castanha de sapucaia (Lecythis pisonis Cambess Lecythidaceae). Revista de Ciências Agro-Ambientais, v.5, n.1, p.111-116, 2007. Disponível em: http://www.unemat.br/revistas/rcaa/docs/vol5/11_artigo_v5.pdf.;

BUTTURI, W.; NUNES, E.J.S.; SILVA, E.P. Banco de dados geográfico aplicado ao cadastro ambiental rural do município de Alta Floresta-MT. Revista de Ciências Agro-Ambientais, v.11, n.1, p.1-8, 2013. Disponível em: http://www.unemat.br/revistas/rcaa/docs/vol11-1/1_artigo_rcaa_v11n1a2013.pdf

CAMARGO, F.F.; COSTA, R.B.; RESENDE, M.D.V.; ROA, R.A.R.; RODRIGUES, N.B.; SANTOS, L.V.; FREITAS, A.C.A. Variabilidade genética para caracteres morfométricos de matrizes de castanha-do-brasil da Amazônia Mato-Grossense. Acta Amazônica, v. 40, n. 4, p. 705-710, 2010. Disponível em: http://www.scielo.br/pdf/aa/v40n4/v40n4a10.pdf.

CAMPOS, A.M.; FREITAS, J.L.; SANTOS, E.S.; LIMA E SILVA, R.B. Fenologia reprodutiva de Bertholletia excelsa Bonpl. em floresta de terra firme em Mazagão, Amapá. Biota Amazônia, v.3, n. 1, p. 1-8, 2013. Disponível em: https:// periodicos.unifap.br/index.php/biota/article/view/421. doi: 10.18561/2179-5746.

FARIAS NETO, J.T.; CARVALHO, J.U.; MULLER, C.H.. Estimativas de correlação e repetibilidade para caracteres do fruto de Bacurizeiro. Ciência e Agrotecnologia, Lavras, v. 28, n. 2, p. 300-305, 2004. Disponível em http://www.scielo.br/pdf/ cagro/v28n2/a08.pdf. doi: 10.1590/S1413-70542004000200008.

FREITAS, S.P.; FREITAS-SILVA, O.; MIRANDA, I.C.; COELHO, M.A.Z. Extração e fracionamento simultâneo do óleo da Castanha-do-Brasil com etanol. Ciência e Tecnologia de Alimentos, v. 27, n. Suppl. 1, p. 14-17, 2007. Disponível em: http://www.scielo.br/pdf/cta/v27s1/a02v27s1.pdf. doi: 10.1590/S010120612007000500002. 
GONÇALVES, L.G.V.; ANDRADE, F.R.; MARIMON JUNIOR, B.H.; SCHOSSLER, T.R.; LENZA, E.; MARIMON, B.S. Biometria de frutos e sementes de mangaba (Hancornia speciosa Gomes) em vegetação natural na região leste de Mato Grosso, Brasil. Revista de Ciências Agrárias, v. 36, n.1, p 31-40, 2013. Disponível em: http://www.scielo.mec.pt/pdf/rca/v36n1/v36n1a06.pdf

GUSMÃO, E.; VIEIRA, F. A.; FONSECA JÚNIOR, E.M.; Biometria de frutos e endocarpos de murici (Byrsonima verbascifolia Rich. ex A. Juss.). Cerne, v.12, n.1, p.84-91, 2006. Disponível em: http://www.redalyc.org/pdf/744/ 74412110.pdf.

KÖPPEN, W. Climatologia con un estudio de los climas de la Tierra. México, 1948. $478 \mathrm{p}$.

LORRENZI, H. Árvores brasileiras: manual de identificação e cultivo de plantas arbóreas nativas do Brasil. $3^{\mathrm{a}}$ ed. Nova Odessa: Instituto Plantarum, 2000. 373p.

MACEDO, M.C.; SCALON, S.P.Q.; SARI, A.P.; SCALON FILHO, H.; ROSA, Y.B.C.J.; ROBAINA, A.D. Biometria de frutos e sementes e germinação de Magonia pubescens ST. Hil (sapindaceae). Revista Brasileira de Sementes, v. 31, n.2, p.202-211, 2009. Disponível em: http://www.scielo.br/pdf/rbs/v31n2/v31n2 a24.pdf.

MATOS, F.S.; NUNES, Y.R.F.; SILVA, M.A.P.; OLIVEIRA, I.S.; Variação biométrica de diásporos de buriti (Mauritia flexuosa L.f. - Arecaceae) em veredas em diferentes estágios de conservação. Ciência Florestal, v.24, n.4, p. 833-842, 2014. Disponível em: http://www.scielo.br/pdf/cflo/v24n4/0103-9954-cflo-24-04-00833.pdf. doi: 10.5902/1980509816583.

MAUÉS M. M. Reproductive phenology and pollination of the brazil nut tree (Bertholletia excelsa Humb. \& Bonpl. Lecythidaceae) in Eastern Amazonia. In: KEVAN P; IMPERATRIZ F. (Eds.). Pollinating Bees - The Conservation Link Between Agriculture and Nature. Brasília: Ministério do Meio Ambiente, 2002, p. 245-254.

SANTOS, F.S.; PAULA, R.C.; SABONARO, D.Z.; VALADARES, J. Biometria e qualidade fisiológica de sementes de diferentes matrizes de Tabebuia chrysotricha (Mart. Ex A. DC.) Standl. Scientia Forestalis, v. 37, n. 82, p.163-173, 2009. Disponível em: http://www.ipef.br/publicacoes/scientia/nr82/cap06.pdf.

SHEPARD JR, G.H.; RAMIREZ, H. Made in Brazil": Human Dispersal of the Brazil Nut (Bertholletia excelsa, Lecythidaceae) in Ancient Amazonia. Economic Botany, v. $65, \quad$ n.1, p.44-65, 2011. Disponível em: http://link.springer.com/article/ 10.1007/s12231-011-9151-6. doi:10.1007/s12231-011-9151-6.

SILVA, R.S,M.; CHAVES, L.J. NAVES, R.V. Caracterização de frutos e árvores de cagaita (Eugenia dysenterica DC.) no sudeste do estado de Goiás, Brasil. Revista Brasileira Fruticultura, v. 23, n. 2, p. 330-334, 2001. Disponível em: http://www.scielo.br/pdf/rbf/v23n2/7976.pdf.

TONINI, H.; COSTA, P.; KAMINSKI, P.E. Estrutura e produção de duas populações nativas de Castanheira-do-brasil (Bertholletia excelsa O. Berg) em Roraima. 
Floresta, v.38, n.3, p.445-457, 2008. Disponível em: http://www.sifloresta.ufv.br/bitstream/handle/123456789/16104/Revista_Floresta_v38 _n3_p445-457_2008.pdf?sequence=1\&isAllowed=y.

ZUFFO, A.M.; ANDRADE, F.R.; ZUFFO JÚNIOR, J.M.; Caracterização biométrica de frutos e sementes de baru (Dipteryx alata Vog.) na região leste de Mato Grosso, Brasil. Revista de Ciências Agrárias, v.37, n.4, p.463-471, 2014. Disponível em: http://www.scielo.mec.pt/pdf/rca/v37n4/v37n4a12.pdf. 